\title{
An avrPto/avrPtoB Mutant of Pseudomonas syringae pv. tomato DC3000 Does Not Elicit Pto-Mediated Resistance and Is Less Virulent on Tomato
}

\author{
Nai-Chun Lin and Gregory B. Martin \\ Boyce Thompson Institute for Plant Research, Tower Rd., Ithaca, NY 14853-1801, and Department of Plant Pathology, \\ Cornell University, Ithaca, NY 14853-4203, U.S.A.
}

Submitted 25 August 2004. Accepted 16 September 2004.

\begin{abstract}
AvrPto and AvrPtoB are type III effector proteins expressed by Pseudomonas syringae pv. tomato strain DC3000, a pathogen of both tomato and Arabidopsis spp. Each effector physically interacts with the tomato Pto kinase and elicits a hypersensitive response when expressed in tomato leaves containing Pto. An avrPto deletion mutant of DC3000 previously was shown to retain avirulence activity on Pto-expressing tomato plants. We developed an avrPtoB deletion mutant of $\mathrm{DC} 3000$ and found that it also retains Pto-specific avirulence on tomato. These observations suggested that avrPto and avrPtoB both contribute to avirulence. To test this hypothesis, we developed an $\triangle a v r P t o \Delta a v r P t o B$ double mutant in DC3000. This double mutant was able to cause disease on a Pto-expressing tomato line. Thus, avrPto and $\operatorname{avrPtoB}$ are the only avirulence genes in DC3000 that elicit Pto-mediated defense responses in tomato. When inoculated onto susceptible tomato leaves and compared with wild-type DC3000, the

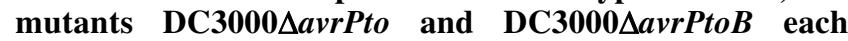
caused slightly less severe disease symptoms, although their growth rate was unaffected. However, DC3000 $\Delta$ avr Pto $\triangle a v r P t o B$ caused even less severe disease symptoms than the single mutants and grew more slowly than them on susceptible leaves. Our results indicate that AvrPto and AvrPtoB have phenotypically redundant avirulence activity on Pto-expressing tomato and additive virulence activities on susceptible tomato plants.
\end{abstract}

The interaction of tomato with the causative agent of bacterial speck disease, Pseudomonas syringae pv. tomato, is a model pathosystem for the study of the molecular mechanisms of gene-for-gene resistance and disease susceptibility (Pedley and Martin 2003). Resistance to speck disease in tomato is conferred by the resistance $(R)$ gene Pto and occurs upon delivery of either of two effector proteins (AvrPto or AvrPtoB) into the plant cell via the bacterial type III secretion system (TTSS) (Kim et al. 2002; Martin et al. 1993; Ronald et al. 1992). Pto encodes a serine-threonine protein kinase and several lines of evidence indicate that the Pto kinase physically

Corresponding author: G. B. Martin; Boyce Thompson Institute; E-mail: gbm7@cornell.edu; Telephone: 607-254-1208; Fax: 607-255-6695.

* The $\boldsymbol{e}$-Xtra logo stands for "electronic extra" and indicates the HTML abstract available on-line contains a supplemental figure not included in the print edition. interacts with the AvrPto and AvrPtoB proteins (Kim et al. 2002; Scofield et al. 1996; Tang et al. 1996). Mutational analysis has revealed that Prf, a protein with a leucine-rich repeat (LRR) and a putative nucleotide binding site (NBS), also is required for Pto-mediated resistance against $P$. syringae pv. tomato (Salmeron et al. 1994, 1996).

In the resistant tomato line Rio Grande-PtoR (RG-PtoR), the Pto haplotype contains Prf and six paralogous Pto genes (LpimPtoA, LpimPtoB/Fen, LpimPtoC, LpimPtoD, LpimPtoE/ Pto, and LpimPtoF) (Riely and Martin 2001), which were introgressed into cultivated tomato (Lycopersicon esculentum) from L. pimpinellifolium (Pedley and Martin 2003). The Pto haplotype in the near-isogenic susceptible line Rio Grande-PtoS (RG-PtoS) is similar, except that there is a deletion of over half of the Pto ortholog (LescPtoE/Pto) due to an unequal crossover event, and LescPtoC is absent due to a second deletion event (GenBank numbers AF220602 and AF220603) (Michelmore and Meyers 1998). Although a Pto ortholog is absent in susceptible L. esculentum lines (e.g., RG-PtoS), there is evidence that one Pto family member, LescPtoF, can induce a hypersensitive response (HR) specifically in the presence of AvrPto and, therefore, might encode a weak recognition determinant (Chang et al. 2002; Riely and Martin 2001). A mutant line, RG-prf3 (in the RG-PtoR background), is available with an approximately $1-\mathrm{kb}$ deletion in the $\operatorname{Prf}$ open reading frame (ORF), which abolishes Pto-mediated resistance against $P$. syringae pv. tomato (Salmeron et al. 1996).

The avrPto gene originally was isolated from $P$. syringae pv. tomato strain JL1065 based on its ability to confer avirulence to normally virulent $P$. syringae pv. tomato strains on Ptoexpressing tomato lines (Ronald et al. 1992). avrPto encodes a small, hydrophilic protein of 164 amino acid residues which is secreted into the plant cell by the $P$. syringae pv. tomato TTSS, where it elicits Pto-specific and Prf-dependent resistance (Pedley and Martin 2003; Salmeron and Staskawicz 1993; van Dijk et al. 1999). The solution structure for AvrPto was solved recently, and found to comprise a three-helix bundle with an $\Omega$ loop that is required for interaction with Pto (Wulf et al. 2004). AvrPto is myristylated and localizes to the host plasma membrane; this localization is required for its avirulence activity (Shan et al. 2000). Although avrPto confers avirulence to normally virulent $P$. syringae pv. tomato strains, surprisingly, deletion of avrPto from P. syringae pv. tomato strains DC3000 or JL1065 did not eliminate the ability of these bacteria to trigger Pto-specific plant resistance (Ronald et al. 1992). This observation suggested that some $P$. syringae pv. tomato strains 
contain a second avirulence gene which is recognized by Pto kinase (Ronald et al. 1992).

A second Pto-specific avirulence gene, avrPtoB, was identified from a cross-kingdom yeast two-hybrid screen using Pto kinase as the bait and a prey library consisting of random genomic fragments from DC3000 (Kim et al. 2002). AvrPtoB (also known as HopAB2) (M. Lindeberg and A. Collmer unpublished data) has 52\% amino acid identity with VirPphA (also known as HopAB1), a type III effector from $P$. syringae pv.

A

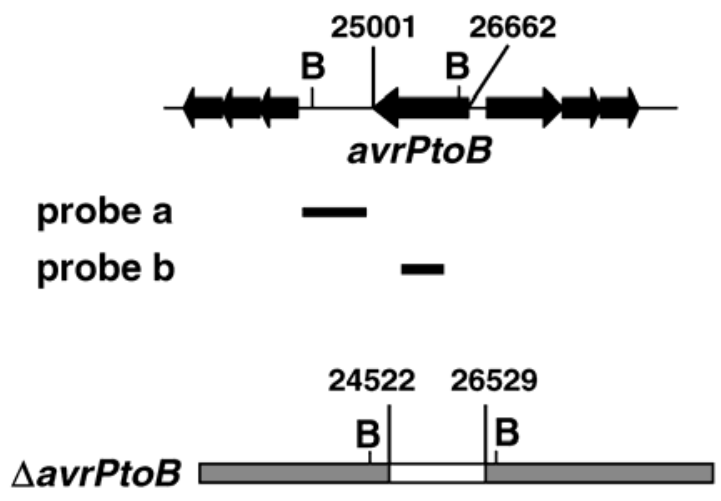

C

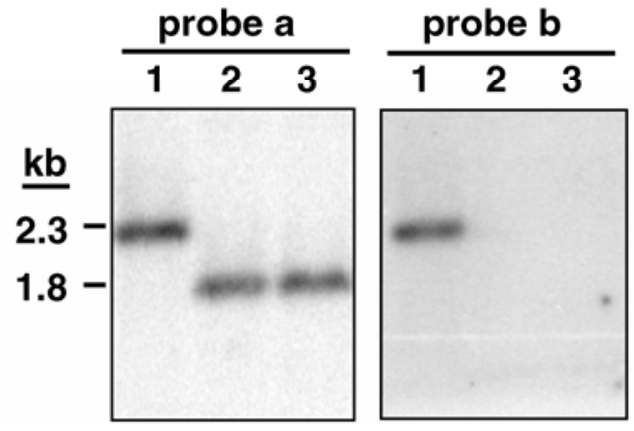

Fig. 1. Development and confirmation of avrPtoB-deletion mutants. A, Arrows indicate direction of transcription, numbers above avrPtoB are the coordinates in the DC3000 genome sequence, and ' $\mathrm{B}$ ' indicates a Bam $\mathrm{HI}$ site. DNA fragments (probes a and b) were used in DNA gel blot analysis for confirmation of the deletion of $a v r P t o B$. B, The region replaced by an nptII gene without a terminator in the $a v r P t o B$-deletion strains is shown as a white box. C, DNA gel blot showing that replacement of avrPtoB by the nptII gene caused expected changes in hybridization to probes a and b. 1 ,

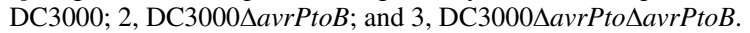

phaseolicola that possesses both avirulence and virulence activities (Jackson et al. 1999). Although AvrPtoB has little similarity to AvrPto at the amino acid sequence level, it is secreted via the TTSS and can elicit Pto-mediated, Prf-dependent resistance in tomato, similar to AvrPto. Similar to observations with avrPto, disruption of $a v r P t o B$ in DC3000 by insertional mutagenesis did not abolish Pto-mediated resistance (Kim et al. 2002).

Many Avr proteins are now known to also have roles in promoting virulence of bacterial strains growing on susceptible plants (Jackson et al. 1999; Lorang et al. 1994; Ritter and Dangl 1995; Swords et al. 1996; Tsiamis et al. 2000). Both AvrPto and AvrPtoB have been shown to have virulence activity when expressed in bacterial strains that do not naturally possess them (Abramovitch et al. 2003; Chang et al. 2000; Jackson et al. 2002; Shan et al. 2000). For example, P. syringae pv. tomato strain $\mathrm{T} 1$ (hereafter T1) grows to higher levels in susceptible tomato leaves and causes more severe disease symptoms when it expressed avrPto from an introduced plasmid. The basis for AvrPto virulence activity has been studied in Arabidopsis spp., where it appears to interfere with host cell-wall defenses (Hauck et al. 2003). AvrPtoB enhances symptom development in a bean pod assay when expressed in P. syringae pv. phaseolicola (Jackson et al. 2002). In addition, AvrPtoB acts as a pathogenicity factor on certain tomato lines and has been demonstrated to act inside the plant cell to inhibit programmed cell death, which likely constitutes one of the mechanisms of its virulence activity (Abramovitch et al. 2003; Jamir et al. 2004).

Activity of individual effectors in their native bacterial strains often is hard to detect by loss-of-function experiments, possibly because it is either subtle or can be complemented by another effector gene with similar function (Alfano and Collmer 2004; Shan et al. 2000; White et al. 2000). Here, we describe the development and characterization of an $a v r P t o B-$ deletion mutant and an avrPto/avrPtoB double mutant. Deletion of $a v r P t o B$ from DC3000 did not affect the avirulence of this strain on a Pto-expressing tomato line as was previously observed with an avrPto deletion strain and an $a v r P t o B$ insertion mutant (Kim et al., 2002; Ronald et al., 1992). These data suggest that AvrPto and AvrPtoB may have redundant avirulence activities in DC3000. Indeed, we found that the avrPto/avrPtoB double mutant no longer elicited Pto-mediated resistance, indicating that avrPto and $a v r P t o B$ are redundant $A v r$ genes in DC3000 and constitute the only two effectors in this strain that elicit Pto-mediated resistance in tomato. Interestingly, avrPto and $a v r P t o B$ also were found to affect DC3000 virulence activity on tomato.

Table 1. Bacterial strains and plasmids

\begin{tabular}{|c|c|c|}
\hline Designation & Relevant characteristics $^{\mathrm{a}}$ & Reference or source \\
\hline \multicolumn{3}{|l|}{ Strains } \\
\hline Escherichia coli $\mathrm{DH} 5 \alpha$ & $\ldots$ & Invitrogen Corp. \\
\hline Escherichia coli TOPO 10 & $\ldots$ & Invitrogen Corp. \\
\hline Pseudomonas syringae pv. tomato & $\ldots$ & \\
\hline DC3000 & Wild type, Rif $^{r}$ & Cuppels 1986 \\
\hline DC3000_avrPto & $\Delta$ avrPto:: $\Omega \mathrm{Sp}^{\mathrm{r}} / \mathrm{Sm}^{\mathrm{r}}, \mathrm{Rif}^{\mathrm{r}} \mathrm{Sp}^{\mathrm{r}} / \mathrm{Sm}^{\mathrm{r}}$ & Ronald et al. 1992 \\
\hline DC3000_avrPtoB & $\Delta a v r P t o B::: n p t I I, \operatorname{Rif}^{\mathrm{r}} \mathrm{Kan}^{\mathrm{r}}$ & This study \\
\hline 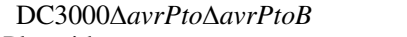 & DavrPto:: $\Omega \mathrm{Sp}^{\mathrm{r}} / \mathrm{Sm}^{\mathrm{r}}$, avvrPtoB:::nptII, $\mathrm{Rif}^{\mathrm{r}} \mathrm{Sp}^{\mathrm{r}} / \mathrm{Sm}^{\mathrm{r}} \mathrm{Kan}^{\mathrm{r}}$ & This study \\
\hline \multicolumn{3}{|l|}{ Plasmids } \\
\hline pavrPtoB & pCPP45 carrying avrPtoB fragment with native promoter, $\mathrm{Tc}^{\mathrm{r}}$ & This study \\
\hline pBluescript II $\left(\mathrm{SK}^{-}\right)$ & $\ldots$ & Stratagene \\
\hline pRK2013 & $\mathrm{Tra}^{+} \mathrm{Mob}^{+} \mathrm{Km}^{\mathrm{r}}$ & Figurski and Helinski 1979 \\
\hline pRK415 & Broad-host-range vector unstable in the absence of selection, $\mathrm{Tc}^{\mathrm{r}}$ & Keen et al. 1988 \\
\hline pCPP45 & Broad-host-range vector with $\mathrm{RP} 4$ par region, $\mathrm{Tc}^{\mathrm{r}}$ & D. W. Bauer, Cornell University \\
\hline pCPP2988 & pBluescriptII SK(-) carrying nptII fragment which lacks terminator, $\mathrm{Amp}^{\mathrm{r}} \mathrm{Kan}^{\mathrm{r}}$ & Alfano et al. 1996 \\
\hline pCR2.1 & TA-cloning vector, $\mathrm{Amp}^{\mathrm{r}} \mathrm{Kan}^{\mathrm{r}}$ & Invitrogen \\
\hline
\end{tabular}

\footnotetext{
${ }^{a}$ Amp: ampicillin; Kan: kanamycin; Tc: tetracycline; Sp: spectinomycin; Sm: streptomycin; Rif: rifampicin.
} 


\section{RESULTS}

Construction of $\triangle a v r P t o B$ and $\triangle a v r P t o \Delta a v r P t o B$ mutants in $P$. syringae pv. tomato $\mathrm{DC} 3000$.

We used marker-exchange mutagenesis in both wild-type DC3000 and DC3000_avrPto (hereafter $\Delta a v r P t o$ ) (Ronald et al. 1992) to delete a 2,007-bp sequence encompassing most of $a v r P t o B$ and a small region downstream (Fig. 1A and B). To accomplish this mutagenesis, plasmid $\mathrm{p} \Delta \mathrm{a} v r P$ to $B$ was generated in the broad-host-range vector pRK415, which is unstable in the absence of tetracycline (Table 1). After triparental mating, the transconjugants were grown on King's medium B (KB medium) containing tetracycline and kanamycin to select for introduction of $\mathrm{p} \triangle \mathrm{avrPtoB}$. Double crossover of the chromosomal regions flanking $a v r P t o B$ resulted in replacement of $a v r P t o B$ by the nptII gene, and the resolved pRK415 carrying full-length $a v r P t o B$ was lost in the absence of tetracycline. As expected, the marker-exchanged mutant in which the double crossover event occurred was kanamycin resistant and tetracycline sensitive. A DNA gel blot confirmed the avrPtoB-deletion mutant in wild-type DC3000 and $\Delta$ avrPto (Fig. 1C). Probe ' $\mathrm{a}$ ', which is a 1.2-kb fragment containing the sequences downstream of avrPtoB, was used to detect the altered BamHI fragment containing avrPtoB sequences. Probe ' $\mathrm{b}$ ', which contains nucleotides 393 to 1,230 of avrPtoB, was used to confirm the deletion of $a v r P t o B$ in the mutants (Fig. 1C). Growth of the DC3000 $\triangle a v r P t o B$ mutant (hereafter $\triangle a v r P t o B$ ) and the DC3000 $\Delta a v r P t o \Delta a v r P t o B$ double mutant (hereafter $\triangle a v r P t o-$ $\triangle a v r P t o B)$ in liquid $\mathrm{KB}$ media containing rifampicin was assayed, and we found that there were no differences in growth rates when compared with wild-type DC3000 (data not shown).

\section{DC3000 $\Delta a v r P t o \Delta a v r P t o B$ is virulent \\ on Pto-expressing tomato leaves.}

We first examined whether the $\triangle a v r P t o B$ mutant was able to elicit disease resistance on the Pto-expressing line Rio GrandePtoR (RG-PtoR). Four days after inoculation, no symptoms of bacterial speck disease were observed on RG-PtoR leaves inoculated with the marker-exchange mutant $\triangle a v r P t o B$ or the previously constructed DC3000 mutant $\triangle \mathrm{avrPto}$, indicating that both strains retain Pto-specific avirulence activity (Fig. 2A). In contrast, the DC3000 strain lacking both avrPto and avrPtoB caused symptoms of bacterial speck disease, indicating that it was no longer avirulent (Fig. 2A). Expression of $a v r P t o B$ from a plasmid in the $\triangle \mathrm{avrPto} \triangle \mathrm{avrPtoB}$ double mutant restored DC3000 avirulence activity. Interestingly, we observed that the double mutant did not cause as severe disease symptoms as wild-type DC3000 does on susceptible tomato lines, suggesting that virulence activity might be affected in the double mutant (see below). Measurements of bacterial numbers in plant leaves were made over a period of 4 days and confirmed the retention of avirulence activity in the single $\triangle a v r P$ to or $\triangle a v r P t o B$ mutants and the loss of avirulence in the $\triangle \mathrm{avrPto} \triangle a v r P t o B$ mutant (Fig. 2B). Interestingly, at day 4 , there was a slight but statistically significant increased level of bacterial growth in the avrPtoB mutant compared with the avrPto mutant (Students' $t$ test; $P=$ 0.018), suggesting that AvrPtoB might have "stronger" avirulence activity than AvrPto. Assays for ion leakage (a measurement of the HR) $4 \mathrm{~h}$ after syringe infiltration of each strain $\left(10^{7}\right.$ $\mathrm{CFU} \mathrm{ml} \mathrm{m}^{-1}$ ) into leaves of RG-PtoR showed that both single mutants caused an HR, whereas the double mutant did not (Fig. $2 \mathrm{C})$. Consistent with the bacterial growth measurements, we observed that ion leakage was greater for $\Delta a v r P t o$ than for $\Delta a v r$ $P t o B$. Together, these results indicate that AvrPto and AvrPtoB are the only two effectors in DC3000 that elicit resistance in Pto-expressing tomato leaves and are largely redundant for this avirulence activity.
A

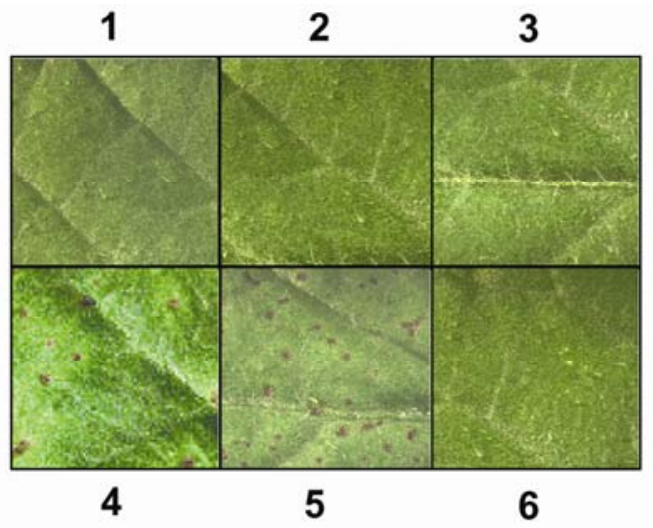

B
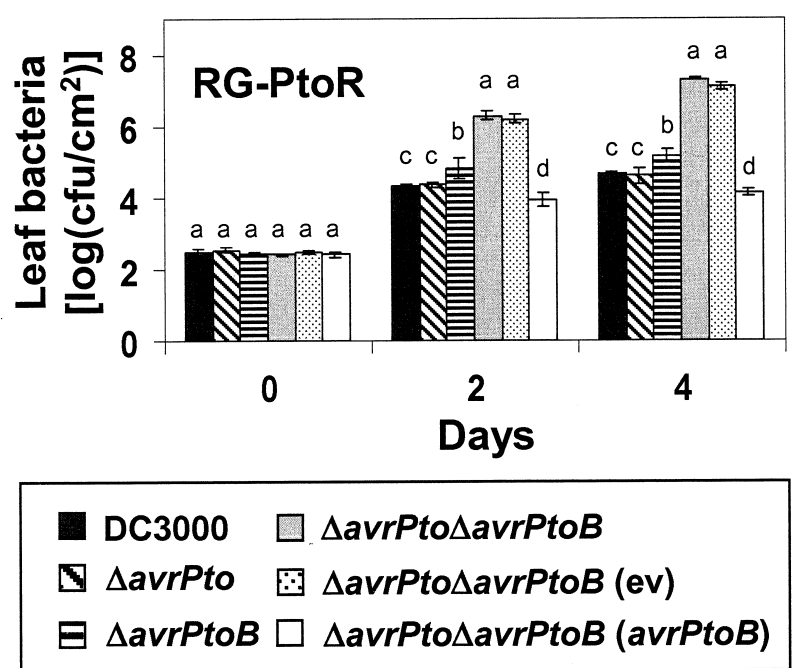

C

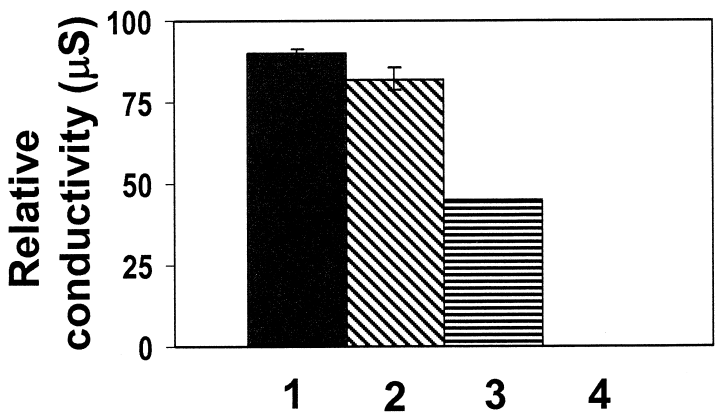

Fig. 2. Assay for Pto-specific avirulence activity of the avrPto and avr$P$ toB single and double mutants. A, Wild-type and mutant forms of DC3000 $\left(10^{4} \mathrm{CFU} \mathrm{m}^{-1}\right)$ were inoculated onto leaves of RG-PtoR (expressing Pto). Photographs of $1-\mathrm{cm}^{2}$ areas were taken through a dissecting microscope 4 days after inoculation of leaves with the following DC3000 strains: 1, DC3000 wild type; 2, $\Delta a v r P t o ; 3, \Delta a v r P t o B ; 4, \Delta a v r P t o \Delta a v r-$ PtoB; 5, $\Delta a v r P t o \Delta a v r P t o B(\mathrm{ev})$; or $6, \Delta a v r P t o \Delta a v r P t o B(a v r P t o B)$. B, Numbers of bacteria in leaves of RG-PtoR $\left(\log \left[\mathrm{CFU} / \mathrm{cm}^{2}\right]\right)$ at 0,2 , or 4 days after inoculation with the strains indicated. Error bars represent the standard deviation of three replicates. Data analysis was performed using Duncan's multiple range test. Means with the same letter assigned above the bars are not different at significance level of 5\%. C, Hypersensitive response (HR) assay. Measurement of ion leakage within the leaf area of RG-PtoR plants infiltrated with the following DC3000 strains $\left(10^{7} \mathrm{CFU}\right.$

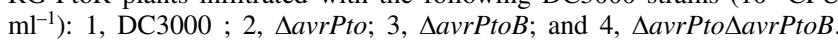
Area infiltrated with inoculation buffer was used for blank control. Relative conductivity $(\mu S)=$ sample conductivity- blank control. Error bars represent the standard deviation of three replicates. The experiments presented in A and B were performed twice with similar results. The HR assay was performed three times with similar results. 
AvrPto and AvrPtoB contribute additively to disease-causing ability of DC3000

on susceptible tomato leaves.

AvrPto is a virulence factor which promotes bacterial growth and disease development when delivered from $P$. syringae pv. tomato $\mathrm{T} 1$ into susceptible plants. In the disease resistance assays described above, we noticed that, although
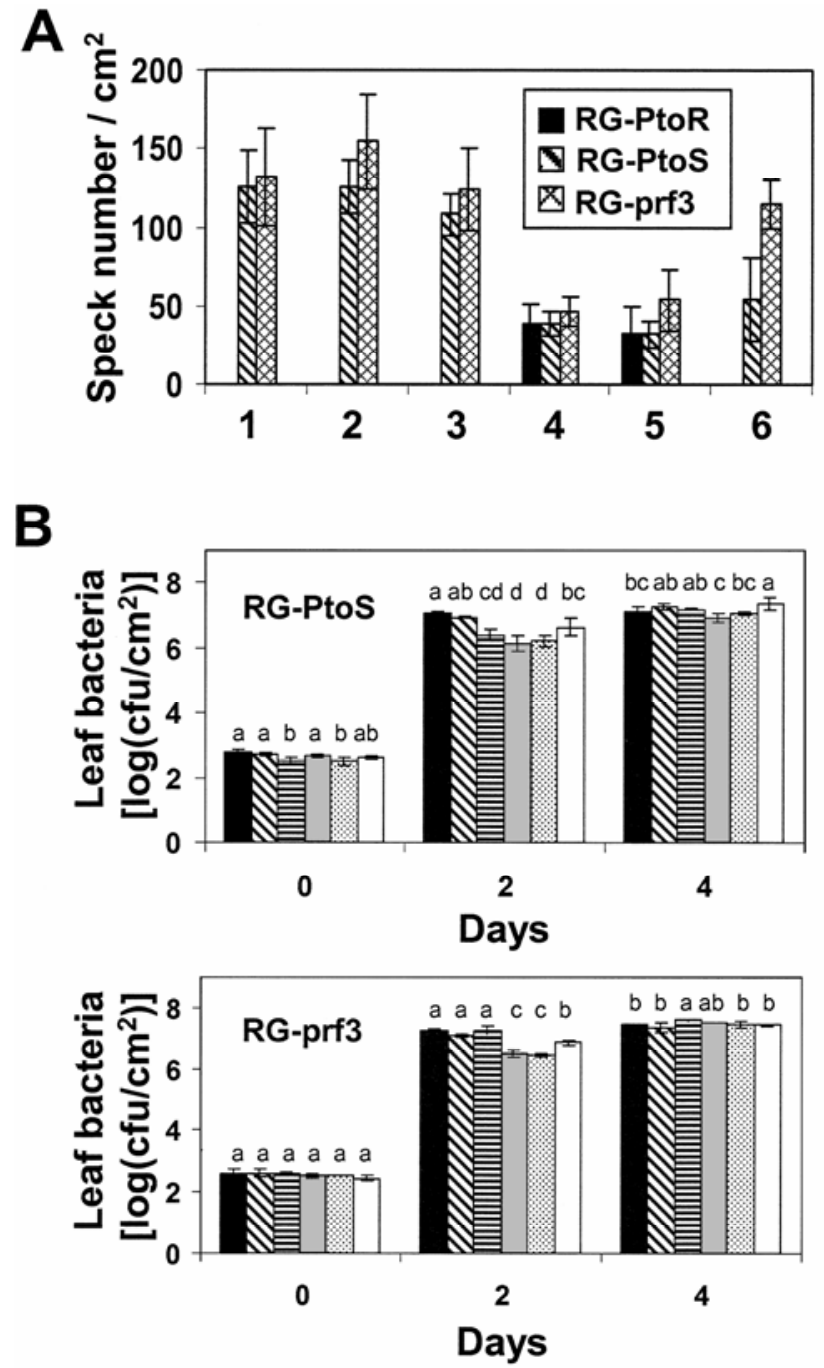

\begin{tabular}{|c|c|}
\hline DC3000 & $\square \Delta$ avrPto $\Delta a v r P t o B$ \\
\hline $\mathbf{\Delta} \Delta$ avrPto & DavrPto $\Delta a v r P t o B(\mathrm{ev})$ \\
\hline E $\triangle$ avrPtoE & $\square$ avrrPto $\Delta$ avrPtoB (avrPtoB) \\
\hline
\end{tabular}

Fig. 3. Ability of DC 3000 and mutant strains to cause disease symptoms and grow on susceptible tomato leaves. A, Speck numbers on leaves of RG-PtoR, RG-PtoS, and RG-prf3 plants 4 days after inoculation with the following DC3000 strains: 1, DC3000 wild type; 2, $\Delta a v r P t o ; 3$, $\Delta a v r P t o B ; 4, \Delta a v r P t o \Delta a v r P t o B ; 5, \Delta a v r P t o \Delta a v r P t o B(\mathrm{ev}) ;$ or 6 , $\triangle a v r P t o \Delta a v r P t o B(a v r P t o B)$. Number of specks per square centimeter was counted from nine independent areas of inoculated leaves. Error bars represent standard deviations. To avoid age-related effects, leaves from the same position of each plant were chosen for counting speck numbers. B, RG-PtoS (upper panel) and RG-prf3 (lower panel). Numbers of bacteria in leaves at 0,2 , or 4 days after inoculation with the bacterial strains are shown. Error bars indicate the standard deviation of populations measured from three 1-cm-diameter leaf discs. Data analysis was performed using Duncan's multiple range test. Means with the same letter assigned above the bars are not different at significance level of $5 \%$. The experiments presented in this figure were performed twice with similar results.
$\Delta \mathrm{a} v r$ Pto $\Delta a v r$ PtoB caused disease on RG-PtoR plants, it was not as severe as DC3000 on susceptible tomato plants. This observation raised the possibility that avrPto and avrPtoB both contribute to virulence activity. In order to test this possibility, we examined the response of two susceptible $L$. esculentum lines, RG-PtoS and RG-prf3, to each of the DC3000 mutant strains. RG-PtoS lacks a functional Pto kinase, whereas RG-prf3 has a deletion in the Prf gene, leading to disruption of Prf function, which is required for Ptomediated resistance.

Leaves of each tomato line were inoculated with the various DC3000 strains and symptoms were scored 4 days later. We consistently observed a reduction in speck symptoms on individual leaves of RG-PtoS and RG-prf3 infected with $\Delta$ avrPto $\triangle \mathrm{a} v r P t o B$ compared with DC3000 or the single mutants. For a more quantitative measurement of these observations, we counted the number of lesions on a random selection of leaves showing discrete specks (Fig. 3A). The number of specks on leaves of RG-PtoS and RG-prf3 infected with the $\Delta a v r P t o$ or $\triangle a v r P t o B$ mutant strains were similar to those on DC3000infected tomato leaves. However, substantially fewer lesion numbers were present on susceptible leaves infected with $\Delta \mathrm{a} v r P t o \Delta \mathrm{a} v r P t o B$. Expression of $a v r P t o B$ from a plasmid in the double mutant increased its lesion-forming ability to the level of the single mutant $\Delta a v r P t o$ on RG-prf3 but not on RGPtoS plants.

Although the results described above suggested that AvrPto and AvrPtoB contribute redundantly to virulence activity, it was possible that our measurements of speck number were unable to detect incremental (additive) contributions of each effector. Therefore, we extended our observations to later stages of the disease process (6 days after inoculation with the various DC3000 strains) (Table 2) and developed a scoring system to represent disease severity. A score of 0 indicated that no disease was observed and a score of 5 indicated severe speck symptoms with large areas of leaf necrosis. We observed that mutant strains $\triangle a v r P t o$ or $\triangle a v r P t o B$ each caused less severe disease symptoms on RG-PtoS and RG-prf3 tomato plants compared with DC3000 (Table 2). Therefore, these observations indicate that each effector does contribute additively to DC3000 virulence. In line with this possibility, the double mutant caused much less severe disease symptoms than each of the single mutants on the susceptible tomato lines tested. Expression of avrPtoB from a plasmid in the double mutant restored its ability to cause disease on RG-prf3 to a level similar to the single mutant $\Delta$ avrPto.

\section{AvrPto and AvrPtoB contribute to the ability of DC3000 to grow on susceptible tomato lines.}

To determine whether the differences in lesion number and disease severity observed with the various DC3000 strains were reflected in differences in bacterial growth in planta, we measured the population numbers in leaves of each strain over a 4-day period (Fig. 3B). Two days after infection of tomato lines RG-prf3, populations of $\triangle a v r P t o \Delta a v r P t o B$ were 5- to 10 fold less than the strains DC3000, $\Delta a v r$ Pto, or $\triangle a v r P t o B$ (Fig. $3 \mathrm{~B}$, lower panel). On tomato line RG-PtoS, populations of $\triangle a v r P t o \Delta a v r P t o B$ were 5- to 10 -fold less than the strains DC3000 or $\triangle a v r P t o$ (Fig. 3B, upper panel). Expression of $a v r P t o B$ in strain $\triangle a v r P t o \triangle a v r P t o B$ partially restored its growth to a level of DC3000 or the single mutants. By day 4, bacterial numbers of $\triangle a v r P t o \Delta a v r P t o B$ were at the same level as wild-type DC3000 (Fig. 3B). Our data suggest that the significantly reduced disease symptoms caused by $\Delta a v r P t o \Delta a v r$ $P t o B$ may be due to its slower growth rate compared with DC3000 and the single mutants. 
Broad host range vector carrying avrPtoB is lost from $\triangle a v r P t o \triangle a v r P t o B($ avrPtoB)

during bacterial multiplication in tomato leaves.

In the experiments described above, a stable broad-host-range vector pCPP45 was used to introduce $a v r P t o B$ into $\Delta a v r P$ to $\Delta a v r P t o B . \Delta a v r P t o \Delta a v r P t o B(\mathrm{pavrPtoB})$ caused overall disease symptoms similar to $\triangle a v r P t o$ in RG-PtoS and RG-prf3 tomato leaves (Table 2). However, addition of pavrPtoB to the double mutant did not completely restore its lesion-forming ability in tomato (Fig. 3A). One possible explanation for these results is that $a v r P t o B$ is not expressed as well from a plasmid as from its native location on the chromosome. This phenomenon has been reported for other effector genes (Guttman and Greenberg 2001). Alternatively, pavrPtoB might be lost from $\triangle a v r P t o \Delta a v r P t o B(a v r P t o B)$ when that strain is growing in leaves. To address this latter possibility, we measured the retention of pavrPtoB in $\triangle a v r P t o \Delta a v r P t o B(a v r P t o B)$ in samples isolated over a 4-day period from leaves of each of three tomato lines (Fig. 4). Remarkably, we observed that over $50 \%$ of bacteria recovered from tomato leaves 2 days after inoculation with $\triangle a v r P t o \Delta a v r P t o B(a v r P t o B)$ had lost pavrPtoB. Four days after inoculation, only $20 \%$ of the leaf bacterial population retained the $a v r P t o B$ plasmid (Fig. 4). Loss of the pavrPtoB occurred equally in all genotypes and, therefore, was not due to selection pressure executed by the Pto pathway. Little or no loss of the empty vector was observed from control strain $\Delta a v r P t o \Delta a v r-$ $P t o B(\mathrm{ev})$ over the same period of time. Therefore, our data indicate that loss of the $a v r P t o B$ plasmid, rather than poor expression of $a v r P t o B$ from a plasmid, is probably the reason that the $\Delta a v r P t o \Delta a v r P t o B(a v r P t o B)$ strain does not produce the same speck numbers as $\triangle a v r P t o$.

\section{DISCUSSION}

Previous studies have shown that avrPto and $a v r P t o B$ encode two sequence-unrelated type III effector proteins, which physi- cally interact with Pto in a yeast two-hybrid system. Both trigger a Pto-specific and Prf-dependent cell death in Agrobacteriummediated transient assays (Kim et al. 2002; Ronald et al. 1992; Scofield et al. 1996; Tang et al. 1996). These data, combined with the earlier observation that mutation of avrPto or $a v r P t o B$ alone in DC3000 did not abolish avirulence activity, strongly suggested that these two proteins have redundant avirulence activity in DC3000 (Kim et al. 2002; Ronald et al. 1992). In this study, by using a series of gene-replacement mutants, we demonstrated that avrPto and $a v r P t o B$ each contribute independently and, for the most part, redundantly, to the Pto-specific avirulence of DC3000. It remains a formal possibility that DC3000 expresses other effectors that might be recognized by Pto but whose existence is "masked" by the presence of other effectors (Abramovitch and Martin 2004; Jackson et al. 1999). However, our data indicate that AvrPto and AvrPtoB are the only two effectors that are responsible for the observed Ptospecific avirulence of DC3000. This appears to be the only reported example where a single bacterial strain expresses two effectors that are recognized by a single host $\mathrm{R}$ protein.

Although the two effectors appeared to have mostly redundant avirulence activity, we observed that deletion of avrPto did not affect avirulence activity of DC3000, whereas deletion of $a v r P t o B$ caused a slight loss in avirulence, as shown by an increase in bacterial growth on Pto-expressing tomato leaves (Fig. 2B). In addition, the $\triangle a v r P t o$ mutant elicited a slightly faster HR than the $\triangle a v r P t o B$ mutant as measured by ion leakage assay (Fig. 2C). These observations suggest that AvrPtoB avirulence activity is "stronger" than AvrPto. The basis for this difference is unknown, but it could be due to greater expression of avrPtoB, translocation of more of the AvrPtoB protein into the plant cell, or a more effective activation of the Pto pathway, perhaps because of stronger binding of AvrPtoB to Pto. We will explore these various possibilities in our future characterization of the differences between these two effector proteins.

Table 2. Disease severity observed on whole tomato plants inoculated with DC3000 or the avrPto and $a v r P t o B$ mutants ${ }^{\mathrm{a}}$

\begin{tabular}{|c|c|c|c|c|c|}
\hline Tomato line & DC3000 & $\Delta$ AvrPto & $\Delta$ AvrPtoB & $\Delta$ AvrPto/ $\Delta$ AvrPtoB & $\Delta$ AvrPto/ $\Delta$ AvrPtoB + pavrPtoB \\
\hline RG-PtoR & 0 & 0 & 0 & 2 & 0 \\
\hline RG-PtoS & 5 & 4 & 4 & 2 & 3 \\
\hline RG-prf3 & 5 & 4 & 4 & 2 & 4 \\
\hline
\end{tabular}

a Disease severity was scored 6 days after inoculation by using a disease index scale. The scale combines our data on speck numbers with a subjective visual score for degree of necrosis, as follows: $0=$ no disease; $1=$ average $<20$ specks $/ \mathrm{cm}^{2}$, no confluent necrosis area; $2=$ average between 20 and 100 specks $/ \mathrm{cm}^{2}$, no confluent necrosis area; $3=$ average between 20 and 100 specks $/ \mathrm{cm}^{2}$ with confluent necrosis area; $4=$ average $>100$ specks $/ \mathrm{cm}^{2}$ with confluent necrosis area, $<50 \%$ leaves were wilted; and $5=$ average $>100 \mathrm{specks} / \mathrm{cm}^{2}$ with confluent necrosis area, $>50 \%$ leaves were wilted. This evaluation of disease severity was scored from three independent plants and repeated twice with similar results.

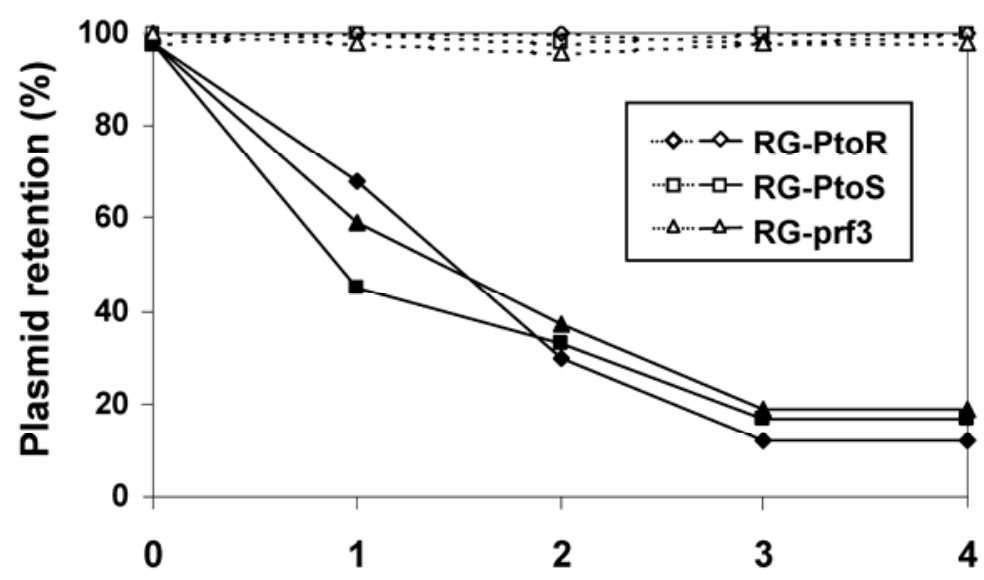

Fig. 4. Measurements of presence of $a v r P t o B$-containing plasmid (pavrPtoB) in DC3000 $a$ avrPto $\Delta a v r P t o B$ (avrPtoB) during growth in tomato leaves. Solid lines show gradual loss of $a v r P t o B$ plasmid in leaves of each tomato genotype. Dashed lines show retention of empty vector, pCPP45, in $\triangle a v r P t o \Delta a v r P t o B$ during growth in tomato leaves. The experiments presented in this figure were performed twice with similar results. 
It often is difficult to demonstrate the virulence function of an effector by loss-of-function assays-probably because they have subtle effects or act redundantly with other effectors (Jackson et al. 1999; Vivian and Gibbon 1997). In our experiments, we observed that the presence of either AvrPto or AvrPtoB was sufficient to sustain lesion-forming ability of DC3000 on tomato lines lacking a functional Pto pathway. However, deletion of both effectors led to fewer lesion numbers (Fig. 3A). Furthermore, the two genes appeared to act additively to enhance overall disease severity caused by DC3000 on tomato (Table 2). Expression of AvrPto in P. syringae pv. tomato $\mathrm{T} 1$ has been demonstrated previously to cause increased bacterial growth and more severe disease symptoms (Chang et al. 2000; Shan et al. 2000). Similar experiments using strain $\mathrm{T} 1$ are not possible with $\operatorname{avrPtoB}$ because, for unknown reasons, this effector does not function in T1 (Kim et al. 2002). However, a role for avrPtoB in virulence (pathogenicity) has been demonstrated by expressing it in a certain DC3000 mutant where it allowed increased bacterial growth in leaves (Abramovitch et al. 2003).

AvrPto has been reported to suppress cell-wall-based defenses in Arabidopsis thaliana and AvrPtoB is known to act broadly as a suppressor of host cell death (Abramovitch et al. 2003; Hauck et al. 2003; Jamir et al. 2004). Our present experiments do not allow us to distinguish between the possibilities that these effectors either act in concert to enhance virulence by targeting the same host process or, instead, act by targeting different host processes. Of course, it is possible that the virulence activity of these effectors relies on some common molecular mechanisms, but it also may involve some distinct ones. In this regard, it is interesting to note that none of the AvrPto-interacting (Bogdanove and Martin 2000) proteins isolated previously interact with AvrPtoB, although both AvrPto and AvrPtoB have been reported to suppress programmed cell death or cell-wall-based defenses (Abramovitch and Martin 2004; Abramovitch et al. 2003; Hauck et al. 2003; Jamir et al. 2004; Ponciano et al. 2004).

Our results can be considered in light of the original "guard" hypothesis (van der Biezen and Jones 1998). This hypothesis posits that Pto is an important virulence target of the effectors AvrPto and AvrPtoB (Pedley and Martin 2003). The interaction of either of the effectors with Pto is postulated to be detected by Prf, with the result being activation of disease resistance pathways. The fact that deletion of AvrPto and AvrPtoB in DC3000 leads to a reduction in bacterial growth rate and a decrease in disease severity clearly supports an important role for these effectors in virulence. Because the only confirmed target of these effectors in the plant, at present, is Pto, our data also support the hypothesis that Pto is an important virulence target for AvrPto and AvrPtoB. There are several lines of evidence that argue against the applicability of the guard hypothesis to the Pto system (Pedley and Martin 2003). However, based on Pto overexpression studies, it is likely that Pto does play a role in host basal defense and, therefore, it is a reasonable virulence target for type III effectors. Further studies using mutants and assays able to detect subtle differences in bacterial growth will be needed to critically test whether Pto is truly a virulence target of AvrPto or AvrPtoB.

To date, symptom development and bacterial growth in leaves are the two major phenotypes used to assay for virulence activities of bacterial effector proteins. Accumulating evidence suggests that there is not always a correlation between these two phenotypes. For example, mutation of gacS in $P$. syringae pv. syringae $\mathrm{B} 728$ a abrogated the ability of this strain to cause brown spot disease in a growth chamber assay, although it had no effect on its ability to grow in bean leaves (Willis et al. 1990). Similar phenotypes have been observed with two other mutants in $P$. syringae pv. tomato and Xanthomonas campestris pv. malvacearum, which caused reduced disease symptoms but had no effect on bacterial growth (Badel et al. 2003; Yang et al. 1994, 1996). However, although we did not observe differences in bacterial populations 4 days after infiltration with our DC3000 strains, $\triangle a v r P t o \Delta a v r P t o B$ did have smaller populations compared with DC3000 and single mutants at 2 days after inoculation. Therefore, the slower growth rate of $\triangle a v r P t o \Delta a v r P t o B$ in susceptible tomato leaves may account for the reduction in lesion formation and, ultimately, disease severity.

It remains unknown how AvrPto and AvrPtoB might alter host processes to promote disease development in susceptible tomato lines. Although AvrPtoB acts as a suppressor of programmed cell death, we did not observe significant differences in lesion size or speck numbers caused by DC3000 and $\Delta a v r P t o B$. However, we observed more confluent necrotic areas on leaves infiltrated with DC3000, $\Delta a v r P t o$, and $\Delta a v r$ PtoB than with $\triangle a v r P t o \Delta a v r P t o B$, suggesting that these two effectors might have additional functions involved in the secondary stage of symptom development involving the spread of chlorosis and necrosis from the primary infection sites (O'Donnell et al. 2003a). A possible clue about the role of these effectors might come from studies of ethylene mutants. Analysis of ethylene-insensitive tomato and Arabidopsis plants has revealed an important role of ethylene in both compatible and incompatible interactions (Ciardi et al. 2000; Lund et al. 1998; O'Donnell et al. 2003b). In tomato, the mutant Never ripe $(\mathrm{Nr})$, which is impaired in ethylene perception, develops reduced disease symptoms upon infection with $X$. campestris pv. vesicatoria or DC3000. The reduction of foliar disease development in $\mathrm{Nr}$ plants was not due to reduced bacterial growth, and was a specific effect of ethylene insensitivity (Lund et al. 1998). This phenotype is similar to what we observed on tomato leaves infected with $\Delta a v r$ Pto $\Delta a v r P t o B$. Thus, we hypothesize that one of the virulence activities of AvrPto and AvrPtoB in tomato might act to increase ethylene production or to enhance ethylene sensitivity and, thereby, enhance foliar disease symptoms. In the future, we plan to investigate the possible role of ethylene in the host response to AvrPto and AvrPtoB.

It is a common strategy to use broad-host-range vectors for studies of gene function in phytopathogenic bacteria. Nevertheless, problems associated with loss of plasmids have been reported in studies of $r p o N$ and the $h r p / h r c$ cluster (Fouts et al. 2003; Guttman and Greenberg 2001; Hendrickson et al. 2000). We noticed that ectopic expression of avrPtoB in $\triangle a v r P t o \Delta a v r P t o B$ did not fully restore to $\triangle a v r P$ to levels the lesion-formation ability in tomato lines RG-PtoS or RG-prf3 (Fig. 3A). Investigation of plasmid retention rates revealed that pavrPtoB was quickly lost from $\triangle a v r P t o \Delta a v r P t o B$ recovered from tomato leaves, whereas the empty vector itself was maintained in this strain. This loss of pavrPtoB from $\triangle a v r P t o$ $\triangle a v r P t o B$ in leaves of both resistant and susceptible tomato lines suggests that there might be a cost in fitness for expression of $a v r P t o B$ that is independent of Pto. In addition, it suggests that plant responses toward pathogen attack are elicited either at the very early stage or by a small amount of effector proteins.

In summary, we have used a series of DC3000 mutants to study the effect of AvrPto and AvrPtoB on avirulence and virulence activity in tomato. We found that the effectors have mostly redundant avirulence activity but additive virulence activities. Whether AvrPto and AvrPtoB have entirely different or partially overlapping targets for their virulence activity will be an interesting topic for future investigation, and the DC3000 mutants described here will be useful tools in those studies. 


\section{MATERIALS AND METHODS}

Bacterial strains, plasmids, and culture conditions.

The bacterial strains and plasmids used in this study are listed in Table 1. Pseudomonas syringae strains were grown in liquid $\mathrm{KB}$ medium supplemented with appropriate antibiotics at $30^{\circ} \mathrm{C}$ (King et al. 1954). The $P$. syringae pv. tomato strains used for disease assays were grown overnight in liquid $\mathrm{KB}$ medium with appropriate antibiotics (listed in Table 1). Bacterial cells were harvested, washed with $10 \mathrm{mM} \mathrm{MgCl}{ }_{2}$ twice,

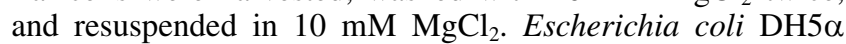
(Invitrogen, Carlsbad, CA, U.S.A.) was used for plasmid maintenance and was grown in LB medium (Hanahan 1985; Sambrook et al. 1989) at $37^{\circ} \mathrm{C}$. The concentrations of antibiotics used in the selective media were as follows: ampicillin, $100 \mu \mathrm{g} / \mathrm{ml}$; kanamycin, $50 \mu \mathrm{g} / \mathrm{ml}$; rifampicin, $100 \mu \mathrm{g} / \mathrm{ml}$; spectinomycin, $50 \mu \mathrm{g} / \mathrm{ml}$; streptomycin, $50 \mu \mathrm{g} / \mathrm{ml}$; and tetracycline, $10 \mu \mathrm{g} / \mathrm{ml}$.

\section{DNA manipulation.}

DNA manipulations and polymerase chain reaction (PCR) were performed using standard protocols (Sambrook et al. 1989). Plasmid DNA was isolated using Wizard Miniprep kits (Promega Corp., Madison, WI, U.S.A.) or Midiprep Plasmid DNA isolation kits (Qiagen, Valencia, CA, U.S.A.). Oligonucleotide primers for sequencing and PCR were obtained from Integrated DNA Technology (Coralville, IA, U.S.A.). PCR was performed with either Taq or Pfu polymerases (Stratagene, La Jolla, CA). Sequencing was performed at Boyce Thompson Institute for Plant Research (Ithaca, NY, U.S.A.) with an automated DNA Sequencer (model 3100; Applied Biosystems, Foster City, CA, U.S.A.). DNA sequences were analyzed using the DNAStar software package (Madison, WI, U.S.A.).

For DNA gel blot analysis, $2 \mu \mathrm{g}$ of genomic DNA was digested with BamHI overnight, and then separated through a $0.8 \%$ agarose gel. DNA was transferred to a Hybond $\mathrm{N}+$ nylon membrane (Amersham Biosciences, Buckinghamshire, U.K.) by the capillary method. DNA probes were labeled with $[\alpha-$ $\left.{ }^{32} \mathrm{P}\right]$-ATP using Klenow fragment of $E$. coli DNA polymerase (Promega Corp.). Prehybridization and hybridization were performed at $65^{\circ} \mathrm{C}$ for $1 \mathrm{~h}$ and overnight, respectively, in $0.5 \mathrm{M}$ phosphate buffer, pH 7.2, $1 \mathrm{mM}$ EDTA, 7\% sodium dodecyl sulfate (SDS) and 5\% dextran sulfate. The membrane was washed twice in $2 \times \mathrm{SSC}(1 \times \mathrm{SSC}$ is $0.15 \mathrm{M} \mathrm{NaCl}$ plus 0.015 M sodium citrate) with $0.1 \%$ SDS, twice in $1 \times$ SSC with $0.1 \%$ SDS, and twice in $0.1 \times \mathrm{SSC}$ with $0.1 \% \mathrm{SDS}$ at $50^{\circ} \mathrm{C}(15 \mathrm{~min}$ each time). Hybridization signals were detected using a Storm system model 840 (Molecular Dynamics, Sunnyvale, CA, U.S.A.).

\section{Construction of the $\triangle a v r P t o B$ and $\triangle$ avrPto $\triangle$ avrPtoB mutants in DC3000.}

DNA sequences flanking the $a v r P t o B$ gene were retrieved from the DC3000 genome sequence. A 2.2-kb fragment containing a $2.1-\mathrm{kb}$ upstream region and the first $133 \mathrm{bp}$ of $a v r-$ $P t o B$ was PCR amplified using the primers avrPtoBmut1F (5'GGTACCGGGCAGCAGGTATAGAATGAAA-3'; KpnI site underlined) and avrPtoBmut1R (5'-AAGCTTGCGGCTGAAC CTGCGGACTGT-3'; HindIII site underlined). A 1.2-kb fragment containing the region downstream of $a v r P t o B$ was amplified using the primers avrPtoBmut2F (5'-GATTTCTCGAGGA CCGCGC-CTTTG-3'; XhoI site underlined) and avrPtoBmut2R (5'-TCTAGAGTACCGCAACCGCCGACACC-3'; XbaI site underlined). An nptII gene without a terminator sequence was obtained from pCPP2988 digested with HindIII and XhoI. These three fragments were cloned sequentially into pBluescript II $\left(\mathrm{SK}^{-}\right)$, and the KpnI-XbaI fragment containing the $n p t I I$ gene and the flanking regions was inserted into the broad-host-range vector, $\mathrm{pRK} 415$, to obtain $\mathrm{p} \triangle a v r P t o B$. This construct was introduced into wild-type $P$. syringae pv. tomato DC3000 (indicated as "DC3000" whenever comparison between different DC3000 derivatives is made) and $\triangle a v r P t o$ for marker-exchange mutagenesis to make $\triangle a v r P t o B$ and $\triangle a v r P t o \Delta a v r P t o B \mathrm{mu}-$ tants. The deletion of $a v r P t o B$ was confirmed by DNA gel blot analysis using probes within and outside of the deletion region.

\section{Construction of complementation plasmids.}

A 1.7-kb fragment containing the hrp box from $a v r P t o B$ and the ORF of $a v r P t o B$ was PCR amplified using the primers avrPtoB-hrp (5'-GGTGTGATGGAACTCTTTCCTGCTC-3'; hrp-box underlined) and avrPtoB-R (5'-TCAG-GGGACTATT CTAAAAGC-3') and cloned into pCR2.1 (Invitrogen). The EcoRI fragment from pCR2.1 was cloned into the broad-hostrange vector, $\mathrm{pCPP} 45$, to create pavrPtoB. pavrPtoB was introduced into $\triangle \mathrm{avrPto} \triangle a v r P t o B$ by triparental mating to obtain a

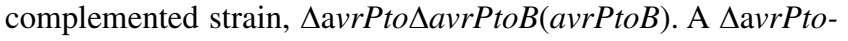
$\triangle a v r P t o B$ strain containing empty vector (hereafter $\triangle a v r P t o-$ $\triangle a v r P t o B(e v)$ ) also was generated as a negative control for

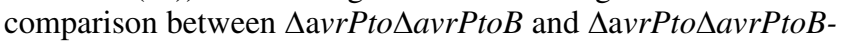
(avrPtoB).

\section{HR assay.}

$\mathrm{HR}$ is represented as the ion leakage within the area infiltrated with the bacterial strains tested. Four hours after infiltration with DC3000, $\Delta \mathrm{avrPto}, \triangle a v r P t o B$, or $\triangle \mathrm{avrPto \Delta avrPtoB}$ at $10^{7} \mathrm{CFU} \mathrm{ml}{ }^{-1}$, four leaf disks $(0.9 \mathrm{~cm}$ in diameter $)$ were collected and incubated in water at room temperature for $1 \mathrm{~h}$. This solution was used to measure sample conductivity with the Acorn series CON5 meter (Oakton Instruments, Vernon Hills, IL, U.S.A.). Leaf disks taken from areas infiltrated with inoculation buffer $\left(10 \mathrm{mM} \mathrm{MgCl}_{2}\right.$ ) were used as blank control to normalize sample conductivity. Ion leakage is presented as the measurement of the conductivity difference between blank control and samples.

\section{Disease assays on tomato.}

Lycopersicon esculentum cv. Rio-Grande (RG) was grown as described previously (Katagiri et al. 2002; Kim et al. 2002). The Rio-Grande lines used in this study include three genotypes: RG-PtoR (Pto/Pto, Prf/Prf), RG-PtoS (pto/pto, PrflPrf; near-isogenic with RG-PtoR), and RG-prf3 (Pto/Pto, prflprf). The RG-prf3 line is derived from RG-PtoR by mutagenesis (Salmeron et al. 1994). Four-week-old tomato plants were vacuum infiltrated with a $P$. syringae pv. tomato suspension containing $1 \times 10^{4} \mathrm{CFU} / \mathrm{ml}$ in $10 \mathrm{mM} \mathrm{MgCl}_{2}$ and $0.002 \%$ Silwet L-77 (Cromptom Co., Middlebury, CT, U.S.A.). After inoculation, plants were monitored every other day for symptom development and bacterial multiplication measurements. Bacterial populations were determined by preparing extracts from leaf tissues collected $1 \mathrm{~h}$ (as day 0 ) and 2 and 4 days after inoculation. At each time point, three 1-cm-diameter leaf disks were collected from three independent plants, and ground in $1 \mathrm{ml}$ of $10 \mathrm{mM} \mathrm{MgCl}$. Bacterial colonies were counted 2 days after plating $10 \mu \mathrm{l}$ from serial dilutions on $\mathrm{KB}$ plates containing rifampicin. From resistant plants, $20 \mu \mathrm{l}$ of undiluted extract was plated (Ronald et al. 1992). Four days after inoculation, speck numbers were counted from nine representative $1-\mathrm{cm}^{2}$ areas which showed discrete lesions (areas with confluent necrosis were avoided). Fully expanded leaflets (usually the third or fourth leaves from the top of the plant) were used for recovery of bacteria and counting of specks. To analyze the rate of loss of pavrPtoB, colonies isolated from leaf samples infected by $\triangle a v r P t o \Delta a v r P t o B(\mathrm{ev})$ and $\triangle a v r P t o \Delta a v r P t o B(a v r P t o B)$ were first cultured on $\mathrm{KB}$ plates containing rifampicin and then 
picked to plates containing tetracycline to select for maintenance of the plasmid.

\section{ACKNOWLEDGMENTS}

This work was supported by National Science Foundation grant number DBI-0077622 (to G. B. Martin), United States Department of Agriculture/National Research Institute grant no. 02-35301-12020 (to G. B. Martin), and Government Scholarship of the Ministry of Education, Taiwan, R. O. C. (to N.-C. Lin). We thank A. Collmer for the kind gifts of pRK415, pCPP45, and pCPP2988; and Y. J. Kim and J. L. Badel for their assistance with marker-exchange mutagenesis and pathogenicity assays.

\section{LITERATURE CITED}

Abramovitch, R. B., and Martin, G. B. 2004. Strategies used by bacterial pathogens to suppress plant defense. Curr. Opin. Plant Biol. 7:356-364.

Abramovitch, R. B., Kim, Y.-J., Chen, S., Dickman, M. B., and Martin, G. B. 2003. Pseudomonas type III effector AvrPtoB induces plant disease susceptibility by inhibition of host programmed cell death. EMBO (Eur. Mol. Biol. Organ.) J. 22:60-69.

Alfano, J. R., Bauer, D. W., Milos, T. M., and Collmer, A. 1996. Analysis of the role of the Pseudomonas syringae pv. syringae HrpZ harpin in elicitation of the hypersensitive response in tobacco using functionally non-polar hrpZ deletion mutations, truncated HrpZ fragments, and hrmA mutations. Mol. Microbiol. 19:715-728.

Alfano, J. R., and Collmer, A. 2004. Type III secretion system effector proteins: Double agents in bacterial disease and plant defense. Annu. Rev. Phytopathol. 42:385-414.

Badel, J. L., Nomura, K., Bandyopadhyay, S., Shimizu, R., Collmer, A., and He, S. Y. 2003. Pseudomonas syringae pv. tomato DC3000 HopPtoM (CEL ORF3) is important for lesion formation but not growth in tomato and is secreted and translocated by the Hrp type III secretion system in a chaperone-dependent manner. Mol. Microbiol. 49:1239-1251.

Bogdanove, A. J., and Martin, G. B. 2000. AvrPto-dependent Pto-interacting proteins and AvrPto-interacting proteins in tomato. Proc. Natl. Acad. Sci. U.S.A. 97:8836-8840.

Chang, J. H., Rathjen, J. P., Bernal, A. J., Staskawicz, B. J., and Michelmore, R. W. 2000. avrPto enhances growth and necrosis caused by Pseudomonas syringae pv. tomato in tomato lines lacking either Pto or Prf. Mol. Plant-Microbe Interact. 13:568-571.

Chang, J. H., Tai, Y.-S., Bernal, A. J., Lavelle, D., T, Staskawicz, B. J., and Michelmore, R. W. 2002. Functional analysis of the Pto resistance gene family in tomato and the identification of a minor resistance determinant in a susceptible haplotype. Mol. Plant-Microbe Interact. 15:281-291.

Ciardi, J. A., Tieman, D. M., Lund, S. T., Jones, J. B., Stall, R. E., and Klee, H. J. 2000. Response to Xanthomonas campestris pv. vesicatoria in tomato involves regulation of ethylene receptor gene expression. Plant Physiol. 123:81-92.

Cuppels, D. A. 1986. Generation and characterization of $\operatorname{Tn} 5$ insertion mutations in Pseudomonas syringae pv. tomato. Appl. Environ. Microbiol. 51:323-327.

Figurski, D., and Helinski, D. R. 1979. Replication of an origin-containing derivative of plasmid RK2 dependent on a plasmid function provided in trans. Proc. Natl. Acad. Sci. U.S.A. 76:1648-1652.

Fouts, D. E., Badel, J. L., Ramos, A. R., Rapp, R. A., and Collmer, A. 2003. A Pseudomonas syringae pv. tomato DC3000 Hrp(Type III secretion) deletion mutant expressing the hrp system of bean pathogen $P$. syringae pv. syringae 61 retains normal host specificity for tomato. Mol. Plant-Microbe Interact. 16:43-52.

Guttman, D. S., and Greenberg, J. T. 2001. Functional analysis of the type III effectors AvrRpt2 and AvrRpm1 of Pseudomonas syringae with the use of a single-copy genomic integration system. Mol. Plant-Microbe Interact. 14:145-155.

Hanahan, D. 1985. Techniques for transformation of E. coli. Pages 109135 in: DNA Cloning: A Practical Approach. D. M. Glover, ed. IRL Press, Oxford.

Hauck, P., Thilmony, R., and He, S. Y. 2003. A Pseudomonas syringae type III effector suppresses cell wall-based extracellular defense in susceptible Arabidopsis plants. Proc. Natl. Acad. Sci. U.S.A. 100:85778582.

Hendrickson, E. L., Guevera, P., and Ausubel, F. M. 2000. The alternative sigma factor RpoN is required for hrp activity in Pseudomonas syringae pv. maculicola and acts at the level of hrpL transcription. J. Bacteriol. 182:3508-3516.

Jackson, R. W., Athanassopoulos, E., Tsiamis, G., Mansfield, J. W., Sesma, A., Arnold, D. L., Gibbon, M. J., Murillo, J., Taylor, J. D., and Vivian, A. 1999. Identification of a pathogenicity island, which contains genes for virulence and avirulence, on a large native plasmid in the bean pathogen Pseudomonas syringae pathovar phaseolicola. Proc. Natl. Acad. Sci. U.S.A. 96:10875-10880.

Jackson, R. W., Mansfield, J. W., Ammouneh, H., Dutton, L. C., Wharton, B., Ortiz-Barredo, A., Arnold, D. L., Tsiamis, G., Sesma, A., Butcher, D., Boch, J., Kim, Y.-J., Martin, G. B., Tegli, S., Murillo, J., and Vivian, A. 2002. Location and activity of members of a family of virPphA homologues in pathovars of Pseudomonas syringae and P. savastanoi. Mol. Plant Pathol. 3:205-216.

Jamir, Y., Guo, M., Oh, H. S., Petnicki-Ocwieja, T., Chen, S., Tang, X., Dickman, M. B., Collmer, A., and Alfano, J. R. 2004. Identification of Pseudomonas syringae type III effectors that can suppress programmed cell death in plants and yeast. Plant J. 37:554-565.

Katagiri, F., Thilmony, R., and He, S. Y. 2002. The Arabidopsis thalianaPseudomonas syringae interaction. In: The Arabidopsis Book. E. M Meyerowitz, ed. American Society of Plant Biologists, Rockville, MD, U.S.A. doi/10.1199/tab.0039. Online publication.

Keen, N. T., Tamaki, D., Kobayashi, D., and Trollinger, D. 1988. Improved broad-host-range plasmids for DNA cloning in gram-negative bacteria. Gene 70:191-196.

Kim, Y.-J., Lin, N.-C., and Martin, G. B. 2002. Two distinct Pseudomonas effector proteins interact with the Pto kinase and activate plant immunity. Cell 109:589-598.

King, E., Ward, M., and Raney, D. 1954. Two simple media for the demonstration of pyocyanin and fluorescin. J. Lab. Clin. Med. 44:301-307.

Lorang, J. M., Shen, H., Kobayashi, D., Cooksey, D., and Keen, N. T. 1994. avrA and avrE in Pseudomonas syringae pv. tomato PT23 play a role in virulence on tomato plants. Mol. Plant-Microbe Interact. 7:508-515.

Lund, S. T., Stall, R. E., and Klee, H. J. 1998. Ethylene regulates the susceptible response to pathogen infection in tomato. Plant Cell 10:371-382.

Martin, G. B., Brommonschenkel, S. H., Chunwongse, J., Frary, A., Ganal, M. W., Spivey, R., Wu, T., Earle, E. D., and Tanksley, S. D. 1993. Mapbased cloning of a protein kinase gene conferring disease resistance in tomato. Science 262:1432-1436.

Michelmore, R. W., and Meyers, B. C. 1998. Clusters of resistance genes in plants evolve by divergent selection and a birth-and-death process. Genome Res. 8:1113-1130.

O'Donnell, P. J., Schmelz, E., Block, A., Miersch, O., Wasternack, C., Jones, J. B., and Klee, H. J. 2003a. Multiple hormones act sequentially to mediate a susceptible tomato pathogen defense response. Plant Physiol. 133:1181-1189.

O'Donnell, P. J., Schmelz, E. A., Moussatche, P., Lund, S. T., Jones, J. B. and Klee, H. J. 2003b. Susceptible to intolerance-A range of hormonal actions in a susceptible Arabidopsis pathogen response. Plant J. 33:245257.

Pedley, K. F., and Martin, G. B. 2003. Molecular basis of Pto-mediated resistance to bacterial speck disease in tomato. Annu. Rev. Phytopathol. 41:215-243.

Ponciano, G., Ishihara, H., Tsuyumu, S., and Leach, J. E. 2004. Bacterial effectors in plant disease and defense: Keys to durable resistance? Plant Dis. 87:1272-1282.

Riely, B. K., and Martin, G. B. 2001. Ancient origin of pathogen recognition specificity conferred by the tomato disease resistance gene Pto. Proc. Natl. Acad. Sci. U.S.A. 98:2059-2064.

Ritter, C., and Dangl, J. L. 1995. The avrRpm1 gene of Pseudomonas syringae pv. maculicola is required for virulence on Arabidopsis. Mol. Plant-Microbe Interact. 8:444-453.

Ronald, P. C., Salmerson, J. M., Carland, F., M., and Staskawicz, B. J. 1992. The cloned avirulence gene avrPto induces disease resistance in tomato cultivars containing the Pto resistance gene. J. Bacteriol. 174:1604-1611.

Salmeron, J. M., Barker, S. J., Carland, F. M., Mehta, A. Y., and Staskawicz, B. J. 1994. Tomato mutants altered in bacterial disease resistance provide evidence for a new locus controlling pathogen recognition. Plant Cell 6:511-520.

Salmeron, J. M., Oldroyd, G. E., Rommens, C. M., Scofield, S. R., Kim, H. S., Lavelle, D. T., Dahlbeck, D., and Staskawicz, B. J. 1996. Tomato Prf is a member of the leucine-rich repeat class of plant disease resistance genes and lies embedded within the Pto kinase gene cluster. Cell 86:123-133.

Salmeron, J. M., and Staskawicz, B. J. 1993. Molecular characterization and hrp dependence of the avirulence gene avrPto from Pseudomonas syringae pv. tomato. Mol. Gen. Genet. 239:6-16.

Sambrook, J., Fritsch, E. F., and Maniatis, T. 1989. Molecular Cloning: A Laboratory Manual. 2nd ed. Cold Spring Harbor Laboratory Press, Cold Spring Harbor, NY, U.S.A.

Scofield, S. R., Tobias, C. M., Rathjen, J. P., Chang, J. H., Lavelle, D. T., Michelmore, R. W., and Staskawicz, B. J. 1996. Molecular basis of gene-for-gene specificity in bacterial speck disease of tomato. Science 274:2063-2065. 
Shan, L., Ping, H., Zhou, J.-M., and Tang, X. 2000. A cluster of mutations disrupt the avirulence but not the virulence function of AvrPto. Mol. Plant-Microbe Interact. 13:592-598.

Swords, K. M. M., Dahlbeck, D., Kearney, B., Roy, M., and Staskawicz, B. J. 1996. Spontaneous and induced mutations in a single open reading frame alter both virulence and avirulence in Xanthomonas campestris pv. vesicatoria avrBs2. J. Bacteriol. 178:4661-4669.

Tang, X., Frederick, R. D., Zhou, J., Halterman, D. A., Jia, Y., and Martin, G. B. 1996. Initiation of plant disease resistance by physical interaction of AvrPto and Pto kinase. Science 274:2060-2063.

Tsiamis, G., Mansfield, J. W., Hockenhull, R., Jackson, R. W., Sesma, A., Athanassopoulos, E., Bennett, M. A., Stevens, C., Vivian, A., Taylor, J. D., and Murillo, J. 2000. Cultivar-specific avirulence and virulence functions assigned to avrPphF in Pseudomonas syringae pv. phaseolicola, the cause of bean halo-blight disease. EMBO (Eur. Mol. Biol. Organ.) J. 19:3204-3214.

van der Biezen, E. A., and Jones, J. D. 1998. Plant disease-resistance proteins and the gene-for-gene concept. Trends Biochem. Sci. 23:454456

van Dijk, K., Fouts, D. E., Rehm, A. H., Hill, A. R., Collmer, A., and Alfano, J. R. 1999. The Avr (effector) proteins HrmA (HopPsyA) and AvrPto are secreted in culture from Pseudomonas syringae pathovars via the Hrp (type III) protein secretion system in a temperature- and pH-sensitive manner. J. Bacteriol. 181:4790-4797.

Vivian, A., and Gibbon, M. J. 1997. Avirulence genes in plant-pathogenic bacteria: Signals or weapons. Microbiology 143:693-704.

White, F. F., Yang, B., and Johnson, L. B. 2000. Prospects for understanding avirulence gene function. Curr. Opin. Plant Biol. 3:291-298.

Willis, D. K., Marabaak, E. M., Rich, J. J., Barka, T. M., Lindow, S. E., and Panopoulos, N. J. 1990. Isolation and characterization of a Pseudomonas syringae pv. syringae mutant efficient in lesion formation on bean. Mol. Plant-Microbe Ineract. 3:149-156.

Wulf, J., Pascuzzi, P. E., Fahmy, A., Martin, G. B., and Nicholson, L. K. 2004. The solution structure of type III effector protein AvrPto reveals conformational and dynamic features important for plant pathogenesis. Structure 12:1257-1268

Yang, Y., De Feyter, R., and Gabriel, D. W. 1994. Host-specific symptoms and increased release of Xanthomonas citri and X. campestris pv. malvacearum from leaves are determined by the $102 \mathrm{bp}$ tandem repeats of pthA and avrb6, respectively. Mol. Plant-Microbe Interact. 7:345-355.

Yang, Y., Yuan, Q., and Gabriel, D. W. 1996. Watersoaking function(s) of $\mathrm{XcmH} 1005$ are redundantly encoded by members of the Xanthomonas avr/pth gene family. Mol. Plant-Microbe Interact. 9:105-113. 